\title{
Super star clusters in the Galactic Center as revealed by HST-NICMOS
}

\author{
Donald F. Figer ${ }^{1}$, Sungsoo S. Kim ${ }^{2}$, Mark Morris ${ }^{1}$, \\ and Eugene Serabyn ${ }^{3}$ \\ ${ }^{1}$ Department of Physics \& Astronomy, University of California, \\ Los Angeles, CA 90095-1562, USA \\ ${ }^{2}$ Korea Advanced Institute of Science and Technology, Department of \\ Physics, Space Science Laboratory, Daejon, 305-701, Korea \\ ${ }^{3}$ Jet Propulsion Laboratory, \\ 171-113, 4800 Oak Grove Drive, Pasadena, CA 91109, USA
}

\begin{abstract}
The three massive clusters in the Galactic Center are not only the most massive young clusters in the Galaxy, but they harbor more Wolf-Rayet stars than any other starburst region in the Local Group. An understanding of their stellar content will be valuable for extending models to starburst regions in other galaxies. We present HST-NICMOS images, luminosity functions, and color-magnitude diagrams of two of these: the Quintuplet and Arches clusters. The images allow the detection of stars over 6 magnitudes fainter than ever before and reveal previously undetected multiple star systems. For the first time, we clearly identify the main sequence in the Quintuplet cluster, and we extend earlier detections of the main sequence in the Arches cluster to $M_{\text {initial }}<10 \mathrm{M}_{\odot}$. We estimate that the Arches cluster has an initial mass function slope which is greater than the Salpeter value. Given their stellar content, the Galactic Center clusters provide both the best nearby examples of super star clusters and the best nearby locale in which to investigate WR phenomena in starburst galaxies and galactic nuclei. We discuss the content of the Galactic Center clusters, with a particular emphasis on how they compare to other massive clusters of the local group. We expect that many of the massive stars in the Galactic Center will soon evolve to become WR stars, and eventually become supernovae at a rate of $\sim 1$ per 20000 years for the next several Myr. We note that our preliminary $\mathrm{N}$-body simulations suggest that such dense clusters are short-lived in the strong tidal field of the Galactic Center, consistent with the fact that no older dense clusters are seen in the central $50 \mathrm{pc}$. This implies a star formation rate of $5\left(10^{-3}\right) \mathrm{M}_{\odot} \mathrm{yr}^{-1}$ in the Galactic Center.
\end{abstract}

\section{Introduction}

Current models for massive starbursts are largely untested by observations of resolved clusters. Even the nearest 'super star cluster', R 136, is so distant that images of its core are hopelessly confused, even with HST. How, then, can starburst models be applied to far away clusters, when we do not know the content of a significant number of massive nearby clusters? Indeed, the situation is much worse considering that the number of massive clusters with known stellar content is much less than the number of factors which are thought to affect their content, i.e., metallicity, cloud temperature, magnetic field, etc. This problem of small number statistics is equally troublesome in extending our 


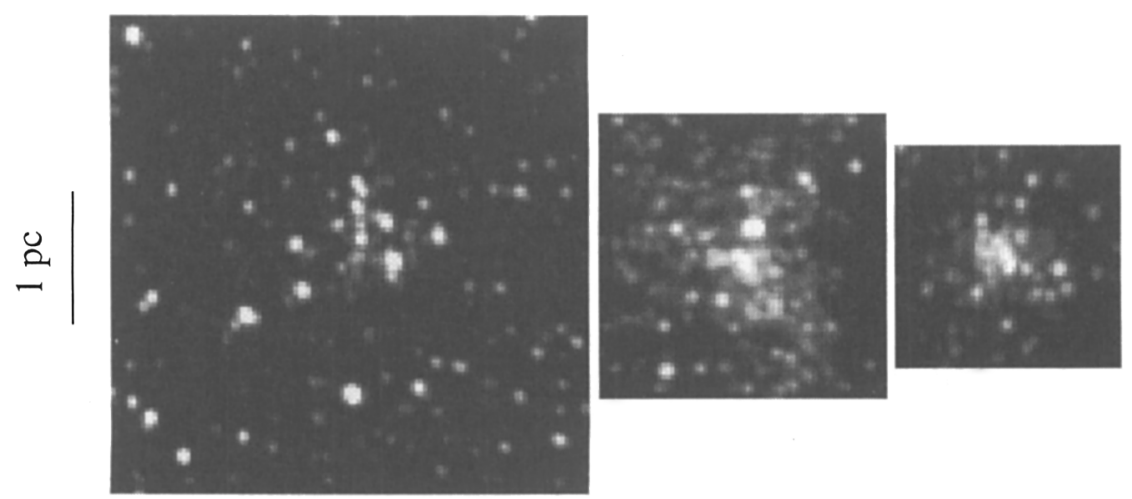

Figure 1. $K^{\prime}$-band images of the Quintuplet (left), Central, and Arches clusters (right). See Figer (1995) for details.

knowledge of nearby Wolf-Rayet stars to Wolf-Rayet galaxies. Recent studies of the massive clusters in the Galactic Center (GC), and their content of WR stars, are making a significant impact in our ability to test star formation and stellar evolution models, thereby providing a test for models which predict the content of starburst regions in other galaxies.

The GC harbors a remarkable sample of young stellar clusters. While occupying just $1 \%$ of the Galactic volume, the central 50 pc contains $10 \%$ of the star formation in the Galaxy (Mezger, Duschl \& Zylka 1996). Correspondingly, the population in the center contains a significant number of the massive stars in the Galaxy, $z 150$ stars with $M_{\text {initial }}>20 \mathrm{M}_{\odot}$. Figure 1 shows a collage of the three extraordinary GC clusters. Each cluster has $\gtrsim 10^{4} \mathrm{M}_{\odot}$ in stars and produces $L>10^{7} \mathrm{~L}_{\odot}$; we collectively refer to them as the 'GC clusters'. The Central cluster was first to be studied, and is now known to contain over 30 stars having $M_{\text {initial }}>20 \mathrm{M}_{\odot}($ Genzel et al. 1996). Serabyn, Shupe, \& Figer $(1998 ; 1999)$ published Keck I images revealing over 100 O-stars packed within 0.6 pc in the Arches cluster (G0.121+0.017), suggesting that this cluster is the densest in the Galaxy. Earlier work revealed a dense collection of emission-line stars with WR-like $K$-band spectra (Nagata et al. 1995; Cotera et al. 1996, 1999). Figer, McLean \& Morris (1999a) published a list of $\sim 30$ stars with $M_{\text {initial }}>20 \mathrm{M}_{\odot}$ in the Quintuplet cluster (G 0.167-0.060), including more bona fide WR stars than in any other Galactic cluster. All three clusters are quite similar in most respects, except for age; the Central and Quintuplet clusters are $\sim 3-5$ Myr old, while the Arches cluster is substantially younger, $\tau_{\text {age }} \lesssim 2 \mathrm{Myr}$.

The GC clusters are even more interesting because they reside in a region with relatively extreme environmental factors which are thought to influence star formation and evolution. The metallicity in the GC may be higher than anywhere else in the Galaxy, impacting both star formation and the evolution of massive stars with strong winds. The ambient magnetic field is 300 times stronger than the average in the Galactic disk, something which is expected to affect protostellar collapse. Morris (1993 and references therein) suggested that the GC environment may alter star formation with respect to that observed in 


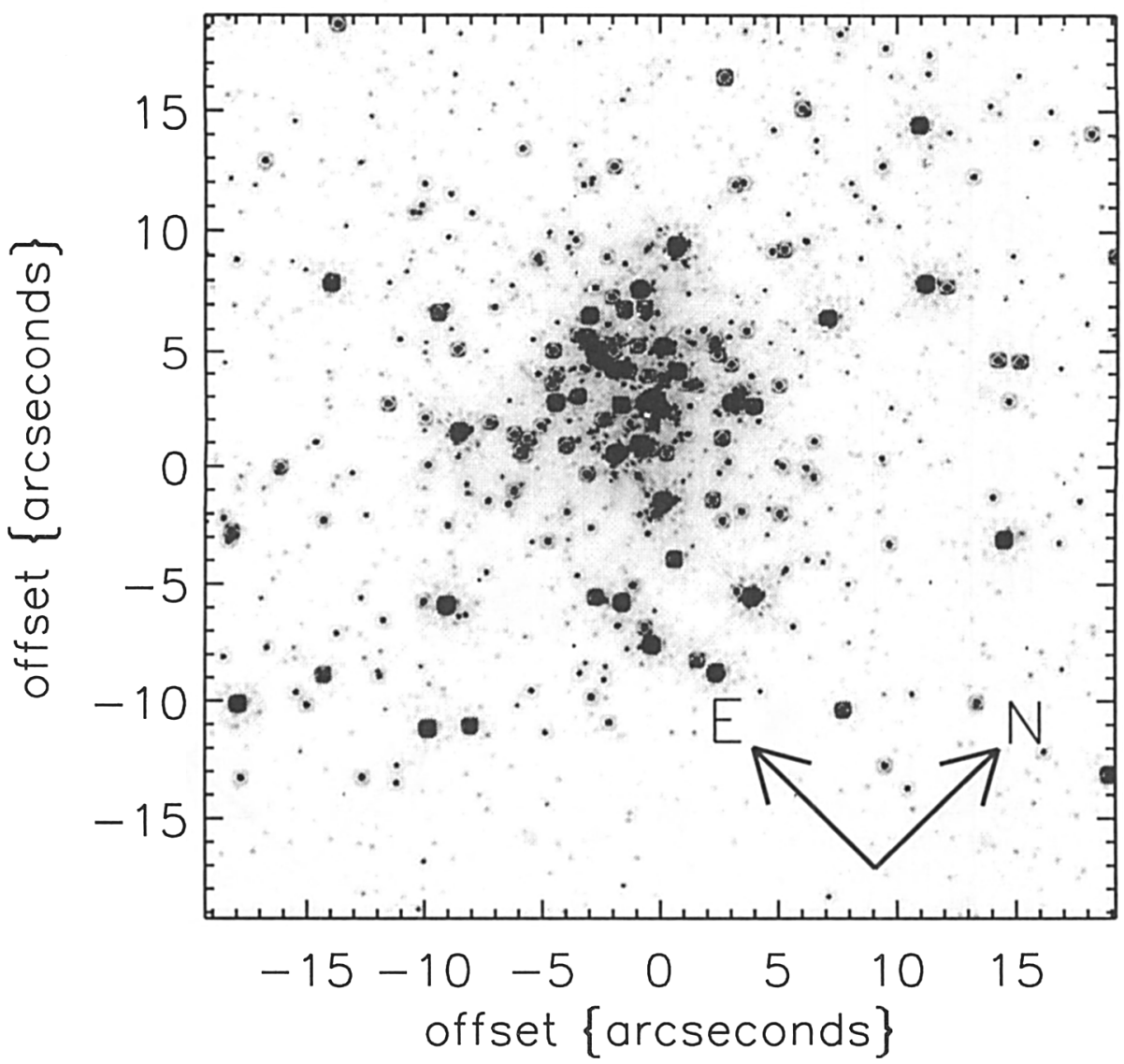

Figure 2. F205W image of the Arches cluster.

the disk of the Galaxy, in particular through a flat initial mass function (IMF) for large masses and by an elevated lower mass cutoff.

In this paper, we review the latest results concerning the GC clusters, with a particular emphasis on our new HST-NICMOS data of the Arches and Quintuplet clusters.

\section{Observations and data reduction}

The Quintuplet and Arches clusters were imaged on UT 1997 September 13-14 in a mosaic pattern using HST-NICMOS in the NIC2 aperture (19'.2 on a side). Four nearby "control" fields for each cluster were also imaged in order to sample the background stellar population. See Figer et al. (1999b) for more details concerning the observations. The images were reduced via the standard NICMOS pipeline (CALNICA, CALNICB; MacKenty et al. 1997).

Figures 2 and 3 show the F205W cluster images $\left(\lambda_{\text {center }}=2.05 \mu \mathrm{m}\right)$. A dense collection of stars can be seen in the Arches cluster, concentrated within a radius of $6^{\prime \prime}$ of the cluster center. The Quintuplet cluster is significantly more 


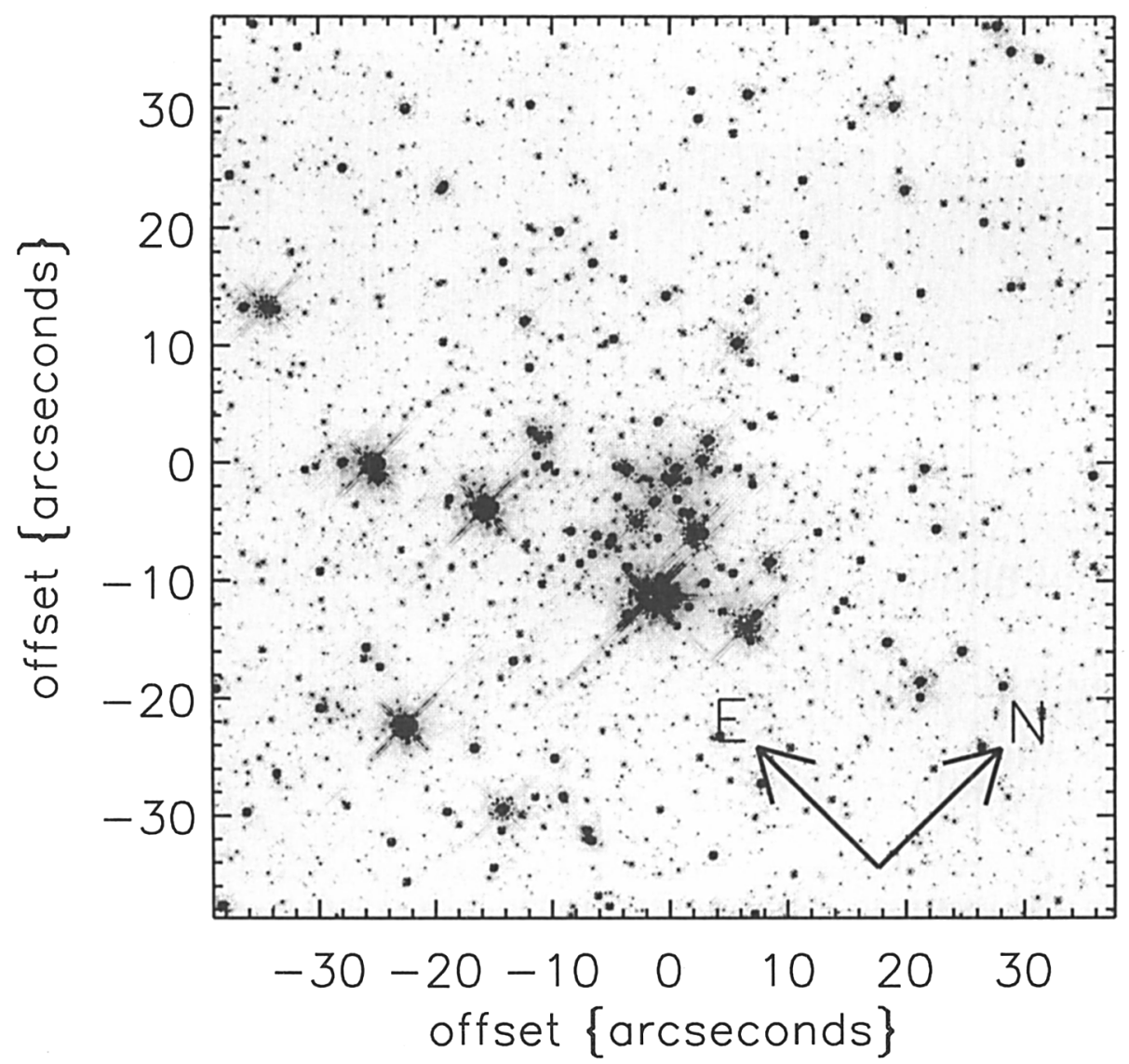

Figure 3. F205W image of the Quintuplet cluster.

spread out. It is difficult to unambiguously distinguish cluster members from the local background population in this image; however, note that the average separation of the massive cluster stars from the centroid of the distribution is about 25", according to Figer et al. (1999a), indicating a region which spans most of the mosaic. The faintest stars which can easily be seen in these figures have $m_{\mathrm{F} 205 \mathrm{~W}} \simeq 19$. Note that two of the original Quintuplet cluster members are multiple ( $\mathrm{qF} \# 192, \mathrm{qF} \# 251, \mathrm{qF} \# 243)$ or extended ( $\mathrm{qF} \# 231$ ), where the nomenclature in parentheses is from Figer et al. (1999a).

We extracted stellar photometry from the images in order to identify main sequences in the clusters by examining the resultant luminosity functions and color-magnitude diagrams. Star-finding, PSF-building, and PSF-fitting procedures were performed using the DAOPHOT-package (Stetson 1987) within the Image Reduction and Analysis Facility (IRAF). See Figer et al. (1999b) for a summary. The amount of crowding in the images differs greatly from image to image. The cluster center regions are extremely crowded such that the detection of stars is limited by diffracted light from neighboring bright stars. The control 


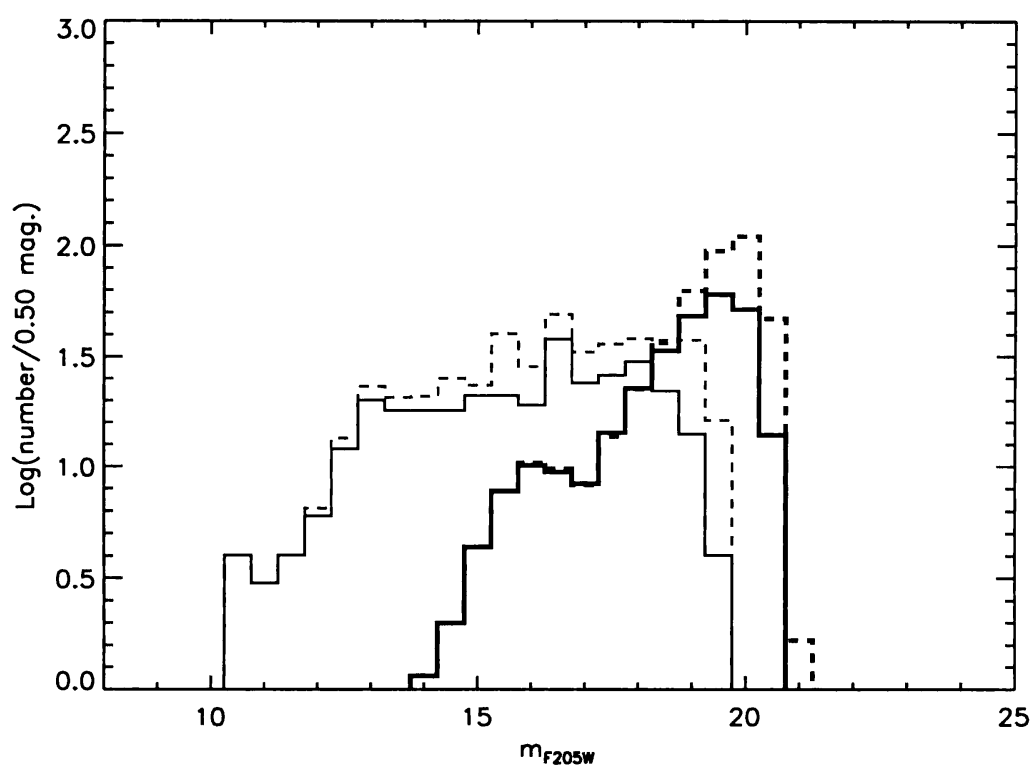

Figure 4. Observed luminosity function for Arches cluster (thin) within a radius of $6^{\prime \prime}$ and nearby control fields (thick) normalized to the area of the cluster. The solid lines represent the observed luminosity function. The dashed lines represent counts after correcting for incompleteness.

fields are moderately crowded by background stars. We attempted to correct the star counts by estimating incompleteness due to crowding (Figer et al. 1999c).

\section{Results}

The extracted luminosity functions are shown in Figures 4 and 5 . The plots for both clusters reveal a shallow function with respect to those for the control fields. This is expected for a reasonable initial mass function for young coeval clusters, in the case of the cluster fields, and for an old population, in the case of the control fields. The sharp falloff at large magnitudes is an expected result of confusion, as opposed to any intrinsic dropoff of faint stars. The peak in counts at $m_{\mathrm{F} 205 \mathrm{~W}} \approx 16$ in the control field luminosity function is due to red giant stars. The higher counts in the Quintuplet cluster field is due to the large size of the mosaic, i.e., the plots are not normalized for surface area; however, note that the control field counts have been normalized to the chosen size of the clusters in both plots.

The color-magnitude diagrams are given in Figures 6 and 7. The plots are restricted to the cores of the clusters, radius $=6^{\prime \prime}$ in the case of the Arches, and radius $=12^{\prime \prime}$ in the case of the Quintuplet. Reddened isochrones, covering $M_{\text {initial }}=0.8-120 \mathrm{M}_{\odot}$ from the Geneva models are overplotted (Meynet et al. 1994). The leftmost isochrone corresponds to an age of $1 \mathrm{Myr}$. The rightmost isochrone corresponds to an age of $10 \mathrm{Myr}$. The models have been reddened for $A_{K}=3.0$. For both clusters, most of the stars fall near $m_{\mathrm{F} 205 \mathrm{~W}}-m_{\mathrm{F} 160 \mathrm{~W}} \simeq 1.5$. 


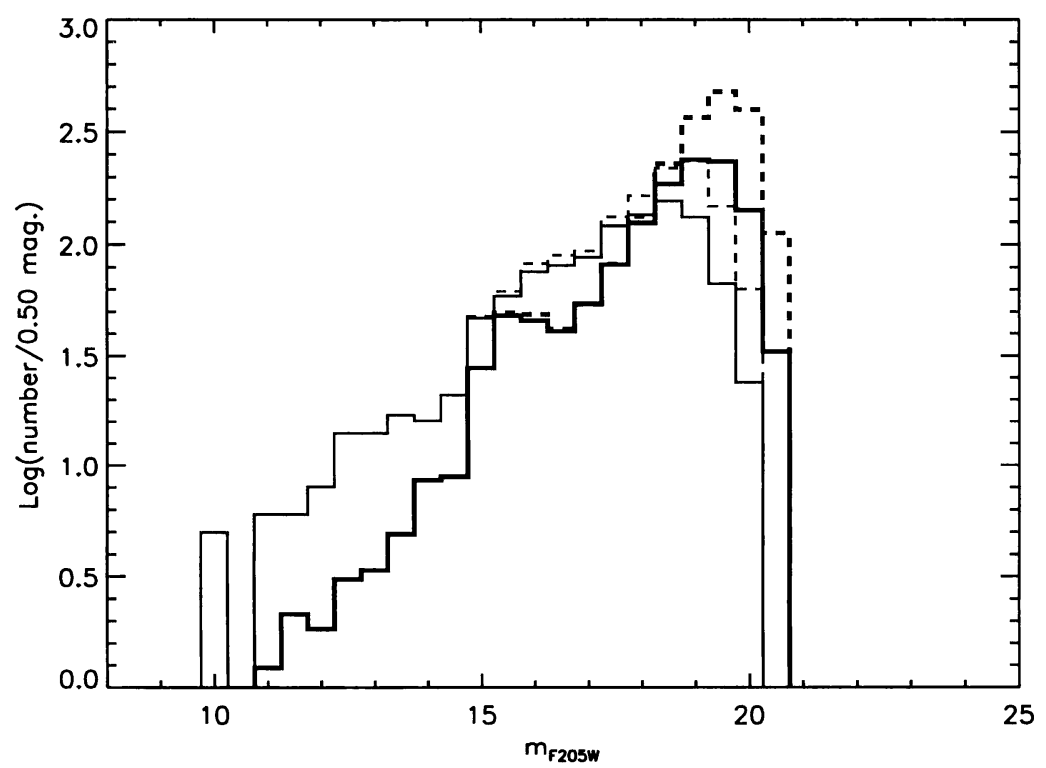

Figure 5. Observed luminosity function for Quintuplet cluster (thin) within a radius of $12^{\prime \prime}$ and nearby control fields (thick) normalized to the area of the cluster. The solid lines represent the observed luminosity function. The dashed lines represent counts after correcting for incompleteness.

The distribution of stars widens at the faint end due to increased photometric errors. The extension of the distribution toward redder colors is due to increased extinction suffered by stars embedded within molecular clouds at the GC.

Figure 8 is a color magnitude diagram for the Quintuplet cluster control fields. A similar diagram can be made for the Arches cluster control fields. The figure shows a striking contrast to those for the cluster fields, in that relatively few stars are brighter than $m_{\mathrm{F} 205 \mathrm{~W}} \simeq 15$. This can be explained by the presence of very old stars in the control fields. We conclude that the cluster fields contain young stars down to fainter than $m_{\mathrm{F} 205 \mathrm{~W}}=16$. This provides the first clear identification of the main sequence in the Quintuplet cluster, and it confirms the earlier identification of the main sequence in the Arches cluster (Serabyn $e t$ al. 1998). We can also see a 'turnoff' in both clusters starting at $m_{\mathrm{F} 205 \mathrm{~W}}=12$ in the Arches cluster and $m_{\mathrm{F} 205 \mathrm{~W}}=13$ in the Quintuplet cluster. This feature is analogous to main sequence turnoffs in older clusters; but massive stars evolve considerably away from the zero age main sequence while still burning hydrogen in their cores, i.e., they are still technically on the main sequence (Meynet et al. 1994).

The difference in the cutoffs in Figures 6 and 7 reflects the difference in ages of the clusters. Note that stars above the turn-off in the Quintuplet cluster span a large range in color, whereas similar stars in the Arches cluster demonstrate the expected trend of redder colors for brighter stars and a blueward trail for the very brightest few stars. The stars above the turnoff in the Arches cluster correspond to the emission-line stars seen by Nagata et al. (1995) and Cotera 


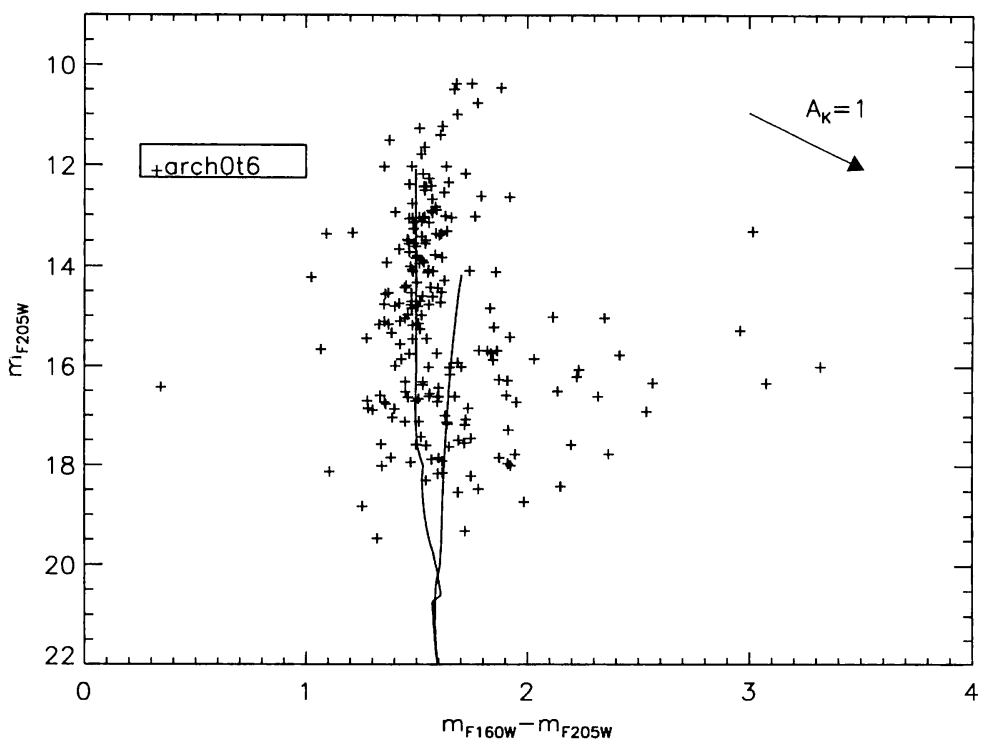

Figure 6. Color-magnitude diagram for the Arches cluster within a radius of $6^{\prime \prime}$ of the cluster center. The color is $m_{\mathrm{F} 160 \mathrm{~W}}-m_{\mathrm{F} 205 \mathrm{~W}}$ and is similar to $H-K$.

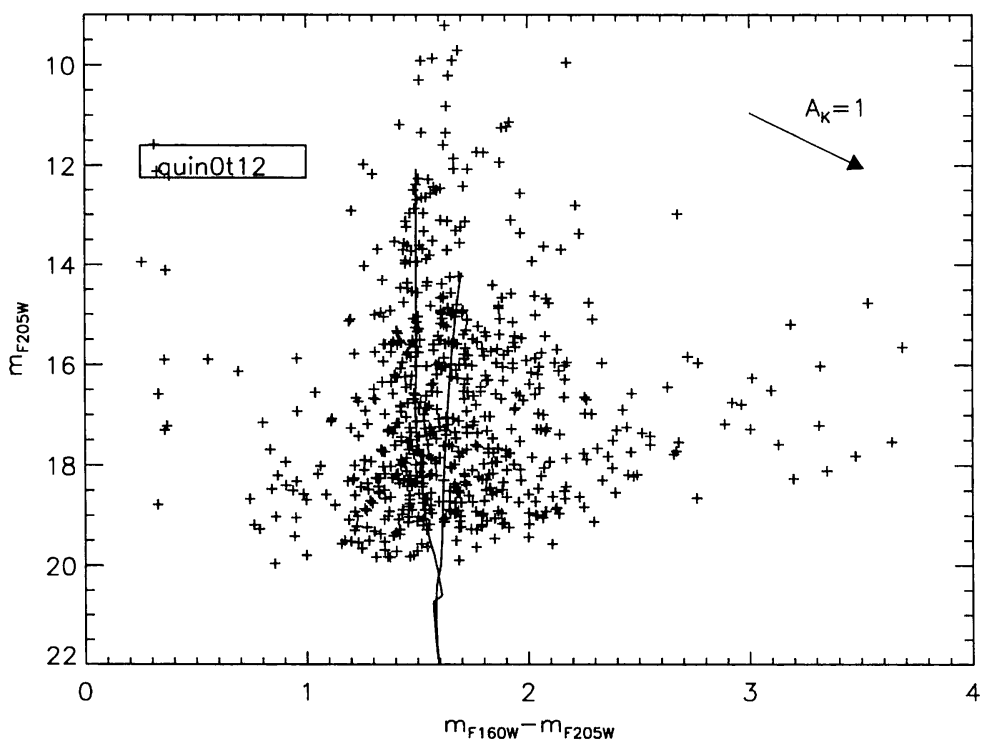

Figure 7. Color-magnitude diagram for the Quintuplet cluster within a radius of $12^{\prime \prime}$ of the cluster center. The color is $m_{\mathrm{F} 160 \mathrm{~W}}-m_{\mathrm{F} 205 \mathrm{~W}}$ and is similar to $H-K$. 


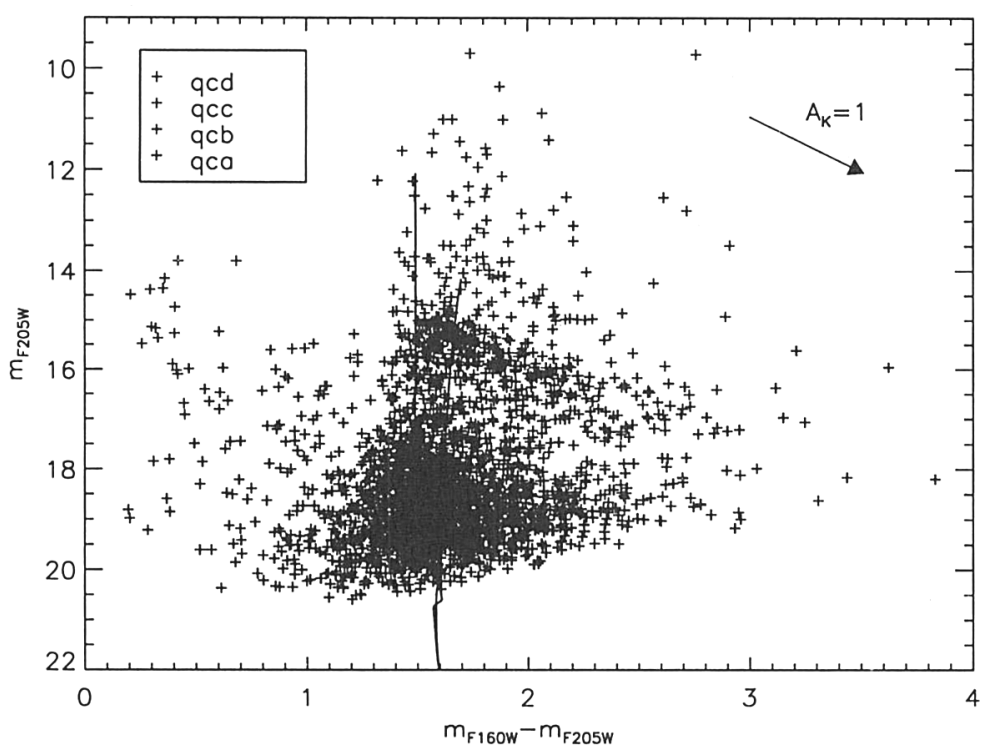

Figure 8. Color-magnitude diagram for the control fields near the Quintuplet cluster. The color is $m_{\mathrm{F} 160 \mathrm{~W}}-m_{\mathrm{F} 205 \mathrm{~W}}$ and is similar to $H-K$.

et al. (1996, 1999); they have strong Paschen- $\alpha$ emission, as seen in our own NICMOS images. These stars are probably still burning hydrogen in their cores and are probably making their first redward excursion in the HR diagram. The stars above the turnoff in the Quintuplet cluster are probably distributed more randomly in color because they are older than the Arches cluster stars, so that some are in the process of making blueward excursions after their first redward excursion in the HR diagram, and some have become much fainter after losing large amounts of mass and becoming WR stars.

The luminosity functions can be transformed into initial mass functions assuming a relation between $m_{\mathrm{F} 205 \mathrm{~W}}$ and $M_{\text {initial }}$. Such a relation depends upon age, metallicity, and the mass-loss rates of stars with strong winds, i.e., the most massive stars. The resultant mass function for the Arches cluster is shown in Figure 9; we assume an age of $2 \mathrm{Myr}$, twice solar metallicity, and the " $2 \times \dot{M}$ "Geneva models for the Arches cluster. The background sample measured in the control fields was subtracted. We limited the sample to an annulus between $3^{\prime \prime}$ and $9^{\prime \prime}$ because the inner core is hopelessly incomplete for all but the brightest stars.

A linear fit has been made through the completeness-corrected data, giving a slope which is shallower than the Salpeter value $(\Gamma=-1.35 ; \mathrm{d}(\log N) / \mathrm{d}(\log m) \propto$ $\Gamma)$. Note that the linear fit is rather poor between $\log \left(M / \mathrm{M}_{\odot}\right)=1.1$ and 1.7. The number of stars in the highest mass bin is misleading. These stars have been put in this bin because they are brighter than stars with $M_{\text {initial }}=120 \mathrm{M}_{\odot}$, where the Geneva models end. 


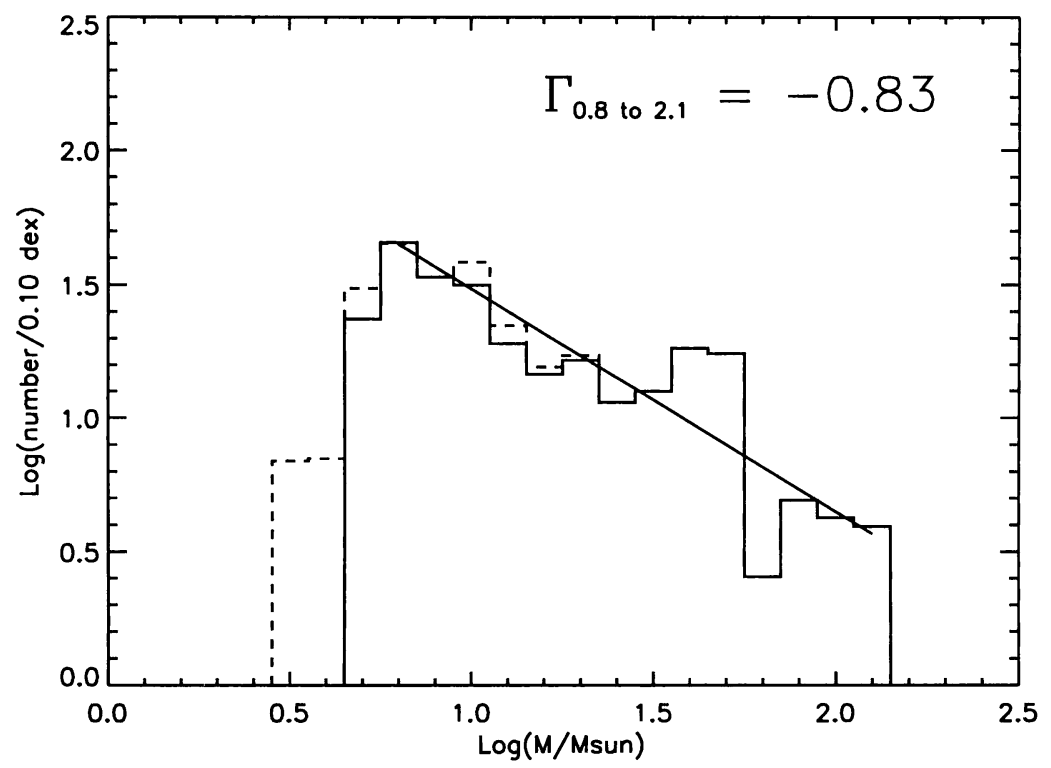

Figure 9. Observed mass function (solid) for Arches cluster in the annulus between $3^{\prime \prime}$ and $9^{\prime \prime}$ of the cluster center. The data have been corrected for incompleteness (dashed), and background counts have been subtracted.

\section{Discussion}

The three GC clusters can be compared to other massive clusters in the Galaxy and Magellanic Clouds. To do this, we estimate total cluster masses using our NICMOS data for the Arches and Quintuplet clusters.

We can make a conservative estimate of the cluster mass by extrapolating the inferred IMF slope to some reasonable lower mass cutoff; the amplitude of the mass function is fixed by the number of stars with a known range of initial masses. We count $\sim 150$ stars with $M_{\text {initial }}>20$ Msun ( $O$-stars) in the Arches cluster. This is a conservative estimate as we are under-counting the number of such stars in the very core of the cluster. Assuming $\Gamma \approx-0.83$ (see Figure 9 ) and $m_{\text {upper }}=120 \mathrm{M}_{\odot}$, we find a total cluster mass of $15000 \mathrm{M}_{\odot}$ for $m_{\text {lower }}=1 \mathrm{M}_{\odot}$, and $19000 \mathrm{M}_{\odot}$ for $m_{\text {lower }}=0.1 \mathrm{M}_{\odot}$. Correspondingly, the total number of stars is 2400 and 17000 . Results of a similar analysis for the Quintuplet cluster will be discussed in Figer et al. (1999c).

How do these clusters compare to other massive clusters, i.e., R 136? Using our new estimates for the Arches cluster, and Table 5 in Figer et al. (1999a), we find that R 136 and the Arches cluster are similar in mass, but that the Arches cluster is about an order of magnitude denser, $\rho_{\text {Arches }} \gtrsim 3\left(10^{5}\right) \mathrm{M}_{\odot} \mathrm{pc}^{-3}$. In fact, the Arches cluster appears to be denser than most globular clusters.

Triggered star formation, such as that induced by cloud-cloud collisions or by supernova shocks, might be more prevalent in the GC than anywhere else in the Galaxy. In fact, the Jean's mass is so large in GC clouds, that triggered star formation might be necessary to form stars (Morris 1993). Of course, many of 
the massive stars in these clusters will become supernovae, thus providing the source of future triggering. Meynet (1995) gives $\sim 5 \times 10^{-7} \times N_{\mathrm{O}}$ supernovae per year during the peak supernova period for a starburst cluster, where $N_{\mathrm{O}}$ is the number of $\mathrm{O}$-stars in the cluster at $\tau_{\text {age }}=0$. These periods are expected to last a few Myr, starting at $\tau_{\text {age }} \approx 4 \mathrm{Myr}$. Using $N_{\mathrm{O}} \approx 100$, we find a supernovae rate of 1 per 20000 years. This assumes that the massive stars do not evaporate below the mass limit for supernovae. Stars in the Quintuplet and Central clusters are closest to the onset of supernovae production, and their massive stars probably number around 100 total. Once the Arches Cluster becomes old enough to participate in supernovae production, the massive stars in the Quintuplet and Central clusters should be gone, so that the total supernova rate in the Galactic Center should remain fairly constant over the next 5 Myr.

It is interesting to consider whether these clusters will become globular clusters. They have masses which are similar to the masses of small globulars (Figer et al. 1999a), but they are subject to the strong tidal field in the GC. In general, a cluster loses its mass by two-body relaxation and stellar evolution (via supernovae and stellar winds), and the dynamical evolution of the cluster is accelerated by the presence of a strong tidal field. The Arches cluster is more compact and younger than the Quintuplet cluster, but otherwise similar in mass. This might suggest that we are watching two different epochs in an evolutionary route of clusters with similar initial conditions. Indeed, FokkerPlanck simulations by Kim et al. (1999) suggest that these clusters disintegrate in less than $10 \mathrm{Myr}$ after birth due to their compactness for $\Gamma>-1.5$. So, while these clusters are similar to other super star clusters, note that they are not proto-globular clusters; they are destined to be ripped apart in a relatively short period of time. This is consistent with the lack of any clusters which are older than $5 \mathrm{Myr}$ in the central $50 \mathrm{pc}$ of the Galaxy. Finally, we note that the number of observed clusters and their lifetimes could give a constraint on the current star formation rate in the GC. Assuming a total mass of $5 \times 10^{4} \mathrm{M}_{\odot}$ in the three GC clusters, and a lifetime of $10 \mathrm{Myr}$, we find a star-formation rate of $5 \times 10^{-3} \mathrm{M}_{\odot} \mathrm{yr}^{-1}$ in these clusters alone.

\section{Conclusions}

We have clearly identified the main sequences in the Quintuplet and Arches clusters down to below $10 \mathrm{M}_{\odot}$, extending our knowledge of the stellar content in these clusters by an order of magnitude in mass. Both clusters show evidence of post main sequence evolution in their color-magnitude diagrams, consistent with the spectral types previously observed. From these diagrams, we confirm previously estimated ages for the two clusters. A preliminary analysis of the mass function indicates that the Arches cluster has an IMF slope which is shallower than the Salpeter value. We find that both clusters will be dispersed by the strong tidal field in the Galactic Center, implying a star-formation rate of $\sim 5 \times 10^{-3} \mathrm{M}_{\odot} \mathrm{yr}^{-1}$.

Acknowledgments. We thank the late Chris Skinner of STScI for providing assistance in performing the NICMOS observations. We thank Christine Ritchie of STScI for assisting in calibrating the NICMOS data. We also thank the NICMOS team for providing assistance in reducing our data. Support for this work was provided by NASA through grant number GO-07364.01- 
96A from the Space Telescope Science Institute, which is operated by AURA, Inc., under NASA contract NAS5-26555. M. Morris acknowledges the Institut d'Astrophysique de Paris for support during this work.

\section{References}

Cotera, A.S., Erickson, E.F., Colgan, S.W.J., Simpson, J.P., Allen, D.A., Burton, M.G. 1996, ApJ 461, 750

Cotera, A.S., Simpson, J.P., Erickson, E.F., Colgan, S.W.J., Burton, M.G., Allen, D.A. 1999, ApJ 510, 747

Figer, D.F. 1995, PhD Thesis, University of California, Los Angeles

Figer, D.F., McLean, I. S., \& Morris, M. 1999a, ApJ 514, 202

Figer, D.F., Morris, M., Kim, S.S., Serabyn, E. 1999b, in: H. Falcke, A. Cotera, W. Duschl, F. Melia, M. Rieke (eds.), The Central Parsecs of the Galaxy, ASP-CS in press

Figer, D. F. et al. 1999c, in preparation

Genzel, R., Thatte, N., Krabbe, A., Kroker, H., Tacconi-Garman 1996, ApJ 472, 153

Kim, S.S., et al. 1999, in preparation

MacKenty, J.W., et al. 1997, NICMOS Instrument Handbook, V2.0 (Baltimore: STScI)

Meynet, G. 1995, A\&A 298, 767

Meynet, G., Maeder, A., Schaller, G., Schaerer, D., Charbonnel, C. 1994, A\&AS 103, 97

Mezger, P.G., Duschl, W.J., Zylka, R. 1996, The A\&A Rev. 7, 289

Morris, M. 1993, ApJ, 408, 496

Nagata, T., Woodward, C.E., Shure, M., Kobayashi, N. 1995, AJ 109, 1676

Serabyn, E., Shupe, D., Figer, D.F. 1998, Nature 394, 448

Serabyn, E., Shupe, D., Figer, D.F. 1999, in: H. Falcke, A. Cotera, W. Duschl, F. Melia, M. Rieke (eds.), The Central Parsecs of the Galaxy, ASP-CS in press

Stetson, P. 1987, PASP 99, 191

\section{Discussion}

Walborn: (1) Where is the Pistol Star located relative to the Quintuplet? (2) If the Arches cluster has 100 O-type stars up to $100 \mathrm{M}_{\odot}$, why don't we see an $\mathrm{H}$ II region in $\mathrm{P} \alpha$ ?

Figer: (1) The Pistol Star is a member of the Quintuplet cluster, but it is not one of the five stars for which the cluster was named. It is located $\sim 20^{\prime \prime}$ south of the cluster center. (2) The medium in the cluster must be relatively evacuated by the O-type stars. The associated H II region is the nearby Thermal Arched Filaments.

Peimbert: Are there observations of the He I recombination lines of the ionized region associated with the clusters?

Figer: I believe that such observations are planned. In addition, ISO observations (PI: Thornley), which may be relevent concerning the excitation of the $\mathrm{H}$ II region, are being reduced. 